${ }^{*}$ Graphical Abstract (for review)

a $\begin{array}{cc}\text { Ground state } & \text { Metastable state } \mathbf{b} \\ \text { (GS) } & \text { (MS) }\end{array}$

(GS)

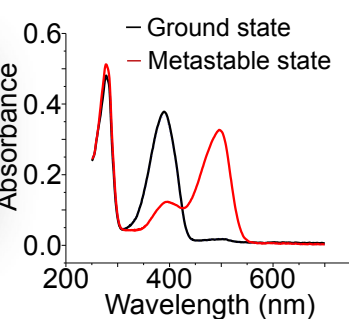

C

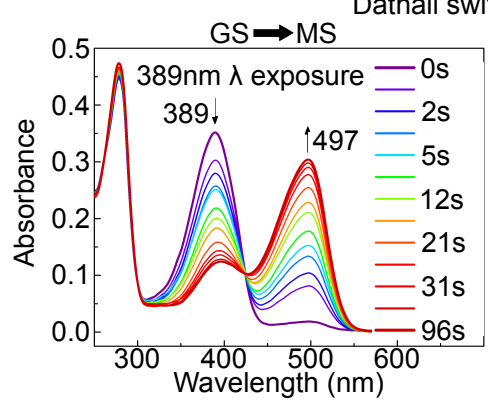

$\mathrm{MS} \rightarrow \mathrm{GS}$

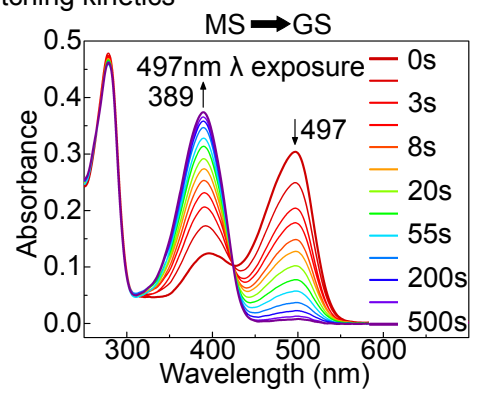

Dathail
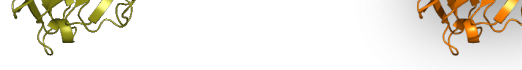

$\Phi=0.0018$

ching kinetics 


\title{
Evolution and characterization of a new reversibly photoswitching chromogenic protein, Dathail
}

\author{
Patricia S. Langan, ${ }^{1}$ Devin W. Close, $1,2,5$ Leighton Coates, ${ }^{3}$ Reginaldo C. Rocha, ${ }^{2}$ Koushik. \\ Ghosh, ${ }^{2}$ Csaba Kiss, ${ }^{1}$ Geoff Waldo, ${ }^{1}$ James Freyer, ${ }^{4}$ Andrey Kovalevsky, ${ }^{3}$ and Andrew R.M. \\ Bradbury. ${ }^{1}$
}

${ }^{1}$ Bioscience Division, ${ }^{2}$ Center for Integrated Nanotechnologies, Los Alamos National Laboratory, NM 87545

${ }^{3}$ Biology and Soft Matter Division, Oak Ridge National Laboratory, TN 37830

${ }^{4}$ Department of Chemical \& Biological Engineering, University of New Mexico, Albuquerque, NM 87131

${ }^{5}$ ARUP Laboratories Institute for Clinical and Experimental Pathology, Salt Lake City, UT

\author{
Abstract \\ We report the engineering of a new reversibly switching chromogenic protein, Dathail. \\ Dathail was evolved from the extremely thermostable fluorescent proteins TGP and \\ eCGP123 ${ }^{1,2}$ using directed evolution and ratiometric sorting. Dathail has two spectrally \\ distinct chromogenic states with low quantum yields, corresponding to absorbance in a \\ ground state with a maximum at $389 \mathrm{~nm}$, and a photo-induced metastable state with an a \\ maximum at $497 \mathrm{~nm}$.: In contrast to all previously described photoswitchable proteins, \\ both spectral states of Dathail are non-fluorescent. The photo-induced chromogenic state \\ of Dathail has a life-time of $\sim 50 \mathrm{~min}$ at $293^{\circ} \mathrm{K}$ and $\mathrm{pH} 7.5$ as measured by UV-Vis


spectrophotometry, returning to the ground state through thermal relaxation. X-ray crystallography provided structural insights supporting a change in conformation and coordination in the chromophore pocket as being responsible for Dathail's photoswitching. Neutron crystallography, carried out for the first time on a protein from the GFP family, showed a distribution of hydrogen atoms revealing protonation of the chromophore 4hydroxybenzyl group in the ground state. The neutron structure also supports the hypothesis that the photo-induced proton transfer from the chromophore occurs through water mediated proton relay into the bulk solvent. Beyond its spectroscopic curiosity, Dathail has several characteristics that are improvements for applications, including low background fluorescence, large spectral separation, rapid switching time and the ability to switch many times. Therefore, Dathail is likely to be extremely useful in the quickly developing fields of imaging and biosensors, including pcFRET, high resolution microscopy, and live tracking within the cell.

\section{Keywords}

Chromoprotein, Photoswitchable, pcFRET, neutron crystallography, ensemble refinement

\section{Introduction}

Fluorescent proteins (FPs) are used as in vivo markers in increasingly sophisticated biotechnological and research applications. FPs with different fluorescent characteristics occur naturally but molecular engineering and directed evolution have increased their variety, improved their properties, and enhanced their utility ${ }^{3-6}$. The first FP, Green Fluorescent Protein (GFP), was isolated from jelly fish Aequoria victoria ${ }^{7}$ and shown by Xray crystallography to be an 11 -stranded $\beta$-barrel enclosing a central chromophore ${ }^{8,9}$. This basic architecture is remarkably conserved across FPs, with variation in spectral properties 
being attributed primarily to the sequence of three amino acids that cyclize and oxidize to form the chromophore, and the local environment in which they are found. In GFP, the mature chromophore (p-hydroxybenzylidenimidazolinone) consists of an imidazolinone ring connected to a planar 4-hydroxybenzyl ring resulting in $\pi$-electron delocalization ${ }^{10}$. The environment of the chromophore - a pocket of side chains and ordered waters that line the interior of the barrel - can alter spectral properties, possibly by influencing chromophore position, protonation state, and electron delocalization ${ }^{4}$.

A useful spectral property, first identified in the Anemonia sulcata chromoprotein asFP595 and its mutants ${ }^{11,12}$, is the ability to convert between fluorescent and nonfluorescent states by irradiation with a specific wavelength of light, often referred to as photoswitching ${ }^{13,14}$. In some cases switching back and forth is possible by alternating exposure wavelengths ${ }^{13}$. In fluorescence microscopy, quickly switching off fluorescence can mitigate saturation and lead to higher image resolution ${ }^{15}$. Further, reversibly photoswitching FPs (RSFP's) can be activated and de-activated during an in vivo process and traced ${ }^{16}$, allowing, for example, the monitoring of protein trafficking within the cell ${ }^{17}$. Although the molecular mechanism for photoswitching is not completely understood, X-ray crystallography has shown that chromophores generally have a cis conformation when fluorescent and a trans conformation when not fluorescent, suggesting the involvement of a cis-trans isomerization ${ }^{18}$ (Schematic 1). Protonation of the chromophore and surrounding amino acids may also play a role, but various studies disagree on the exact mechanism ${ }^{19}$.

Another useful spectroscopic property first identified in FPs isolated from the sea anemone Anemonia sulcata 20 is low fluorescence quantum yield. FPs with quantum yield below 0.001 , referred to as chromoproteins ${ }^{21}$, have a variety of applications, most notably 
as Förster resonance energy transfer (FRET) acceptors $20,22,23$. Chromoproteins have been used to stochastically quench FP-tagged target proteins, thus conferring enhanced resolution in florescence microscopy ${ }^{24}$. A general theme observed for most chromoproteins is that the chromophore adopts the trans conformation whereas it is in the cis conformation for most FPs (Schematic 1). However, there are exceptions to this rule, and trans conformation cannot solely be responsible for the lack of fluorescence.

FPs that possess both of the above-mentioned spectral properties, referred to as reversibly switchable chromoproteins (RSCP), are attracting increasing attention for applications in high-resolution microscopy. For example, the RSCP called Phanta has been used as a non-fluorescent photo-switching acceptor for photochromic Förster resonance energy transfer (pcFRET) and simultaneously functions as part of a biosensor while increasing resolution of the interaction ${ }^{25}$. Despite the increasing variety of RSCPs being developed ${ }^{26}$, applications may be limited by their narrow spectral range, poor spectral overlap with common fluorophores, lack of spectral distinction of their two photo-chromic states, relatively high background fluorescence and low stability through multiple photocycles.

Here, we report the engineering of a new RSCP, referred to as Dathail, named for the Gaelic word for colorful. The protein was evolved from the extremely thermostable fluorescent proteins TGP and eCGP123 1,2 using directed evolution and ratiometric flow cell sorting. Dathail has absorbance in two spectrally distinct states: a ground state with an absorbance maximum at $389 \mathrm{~nm}$, and a photo-induced metastable state with an absorbance maximum at $497 \mathrm{~nm}$. The fluorescence quantum yield on excitation at $488 \mathrm{~nm}$ is extremely low (0.0034) and the initial absorbance from the $497 \mathrm{~nm}$ peak, after fully 
converting to the light-induced state, is $6.1 \%$ of the metastable state. In contrast to all previously described photoswitchable proteins, both spectral states of Dathail are nonfluorescent. These properties suggest that Dathail could be a viable and improved alternative to available RSCPs as a photoswitchable FRET acceptor.

\section{Results}

Directed Evolution. We have previously reported the engineering of two FPs, eCGP123 and TGP, that demonstrate extremely high degrees of thermostability $1,2,27$. eCGP123 $13^{1}$ was developed from an artificial consensus fluorescent protein ${ }^{27}$ by insertional mutagenesis and evolution. Although extremely thermostable, eCGP123 is aggregation-prone, a shortcoming resolved in the derivative protein, Thermal Green Protein (TGP), by surface engineering 2 . In addition to being even more thermostable than eCGP123, TGP is highly soluble and readily crystallizes, making it a particularly useful scaffold for further engineering. The X-ray crystal structures of eCGP123 and TGP were used as scaffolds to guide generation of libraries with randomized side chains. Only amino acid residues within the interior of the $\beta$-barrel that are either in direct, or water mediated, hydrogen bonding distance ( $<3.5$ Angstroms) to the chromophore (Figure 1A-B,E) were mutated. Degenerate libraries were generated and expressed in E. coli, and an iterative strategy using ratiometric flow cytometry sorting and plate imaging was used to identify clones with a variety of spectral properties including red-shifted emission, a spectrum of colors under ambient light (Figure 1C) and, ultimately, proteins identified as being photoswitchable. Sequencing of initial photoswitching clones revealed that mutations localized to a single $\beta$ strand (strand S7; Figures 1 and 2) led to an array of spectral properties. We 
therefore made a degenerate library by modifying residues 140,141, 142, and 144 along this strand to assess how combining different mutations affects photo-switching and spectral properties. Initial libraries were created on the eCGP123 scaffold, but these were transferred to TGP once it became available, due to its improved solubility ${ }^{2}$. This degenerate library resulted in a number of proteins that were isolated based on their ability to photoswitch. Dathail was one of these photo-switching chromoproteins, we chose for further study (Figure 2A).

Spectroscopic Characterization. The UV-Vis absorbance spectrum of Dathail has a pronounced band at 389nm, and a weak band at 497nm (Figure 2B). Upon irradiation with a $389 \mathrm{~nm}$ light source, using a fluorometer, the intensity of the $389 \mathrm{~nm}$ band decreased, while that of the $497 \mathrm{~nm}$ band increased significantly. Saturation occurred at about 100s, at which point the intensities of both absorbance bands ceased to change despite continued illumination. The presence of the isosbestic point at $425 \mathrm{~nm}$ indicates a simple unimolecular photochemical reaction, in which the chromophore undergoes transition from a ground state to a light-induced state through an excited state. Conversion between the ground and the light-induced states could also be obtained by illumination with $405 \mathrm{~nm}$ and $488 \mathrm{~nm}$ wavelength lasers, respectively, normally found in flow cytometers (Figure 2A).

Dathail is similar to Dronpa in that it is negative-switching, i.e. fluorescence is highest in the ground state and is turned off with illumination. Importantly, unlike Dronpa $^{13}$ that is switched off by $488 \mathrm{~nm}$ light, Dathail is switched off by 405 (389) nm light and can be switched back to the ground state with exposure to 488 (497) nm light. This 
characteristic is similar to Padron ${ }^{28}$, but Padron is different in that it is a positive-switching RSFP. Another RSFP called Dreiklang ${ }^{29}$ shows negative-switching with the off state triggered by $405 \mathrm{~nm}$ light, but returns to the on state at a shorter wavelength ( $365 \mathrm{~nm}$ ), suggesting a different mechanism.

Fluorescence emission spectra of Dathail (Supplementary Figure 1A) confirm the existence of two distinct spectral states; the emission of the ground state peaks at $462 \mathrm{~nm}$ $(\mathrm{QY}=0.0034)$ and that of the light-induced state at $514 \mathrm{~nm}(\mathrm{QY}=0.0018)$. Due to their extremely low quantum yields both states can be categorized as chromogenic states, rather than fluorescent states. The residual absorbance at $389 \mathrm{~nm}$ after converting to the lightinduced state is $32.4 \%$ of the initial absorbance value after 500 seconds of exposure. Conversely, the initial absorbance of the light-induced state at $497 \mathrm{~nm}$ is $6.1 \%$ of the ground state absorbance at that wavelength (Figure 2C). Therefore, the absorbance profiles of the two states are easily distinguishable, as the peaks are $108 \mathrm{~nm}$ apart, with relatively low leftover absorbance of each peak after photoswitching (Figure 2B-C). The states can be interchanged for 10 cycles with little change in spectral properties (e.g. retains $90 \%$ decrease in fluorescence, Supplementary Figure 1B). Dathails photostability approaches that of recently developed advanced proteins such as Dreiklang, rsEGFP2, and rsEGFP (this latter protein shows no detectable decrease of fluorescence after 20 cycles and only 15\% decrease after 200 cycles of photoswitching ${ }^{29-31}$. In the forward reaction from the ground state to the light-induced state half of the species are converted within 12 seconds and in the reverse reaction, half are converted within 3 seconds (Figure 2C). In our hands Dronpa has considerably slower kinetics, requiring approximately 10 minutes to switch half the 
species forward, and 3 minutes for the reverse reaction (Supplementary Figure 2). The faster photoswitching kinetics of Dathail may be advantageous in certain applications.

The lifetimes of the photo-induced state as a function of temperature and $\mathrm{pH}$ are summarized in Table 1. The photo-induced state is metastable, returning back to the ground state through thermal relaxation within several hours at $10^{\circ} \mathrm{C}$ and after about a minute at $60{ }^{\circ} \mathrm{C}$. Interestingly, the lifetimes of these two states are dependent on $\mathrm{pH}$. Above $\mathrm{pH}$ 8.5, the metastable state lifetimes do not change with increasing $\mathrm{pH}$, whereas its lifetime drops significantly at $\mathrm{pH} 7.5$. The lifetime increases again at $\mathrm{pH} 6$, with the values surpassing those at higher $\mathrm{pH}$. This indicates that protonation/deprotonation events occurring in Dathail over the 6-10 pH range alter the ionization states of the protein residues. Interestingly, the lifetime is shortest in the physiological pH range, again an advantage of Dathail for biomedical applications. 
X-ray structures of Dathail ground and metastable states. X-ray crystal structures of the ground and metastable states were determined from two isomorphous crystals grown in the same drop. The ground state structure was determined from data collected from one crystal with minimal exposure to light, whereas the metastable state structure was determined from data collected from a second crystal exposed to $405 \mathrm{~nm}$ wavelength light (The crystal was exposed to a $50 \mathrm{~mW}$ laser before cryo-cooling, and also during data collection at $100^{\circ} \mathrm{K}$ ). Ground and metastable state data were collected and processed to the same resolution (1.65 $)$ ), space group and unit cell dimensions (Table 2). Examination of the resulting structures revealed several important structural differences that could be attributed to excitation by the laser.

One of the most striking differences is in the conformation and coordination of the chromophore. In the ground state, the chromophore 4-hydroxybenzylidene group (shown in blue in Figure $3 \mathrm{~A}$ and $\mathrm{B}$ ) is modeled in the cis conformation; the same conformation observed in TGP and other proteins with stable fluorescence ${ }^{2}$. In the metastable state, there is poor density for this group of the chromophore, implying that excitation at $405 \mathrm{~nm}$ leads to a significant conformational change involving movement of the chromophore away from the cis conformation (Figure 3C and D). A non-structured 4-hydroxybenzylidene ring that moves freely within the chromophore cavity could explain the lack of strong electron density for the trans-conformation, as the averaged electron density cannot provide a single location for it.

We investigated this possibility further by using isomorphous difference electron density maps ${ }^{32}$, $\mathrm{F}_{0}\left(\right.$ light, or dark)-Fo(dark or light), and ensemble refinement ${ }^{33}$. Positive (green) electron density in isomorphous difference maps calculated by subtracting 
metastable state observed amplitudes (Fo) from ground state Fo (Fodark-Folight) clearly confirms that the chromophore is more likely to be in the cis conformation in the ground state than the metastable state (Figure 3E). Negative (red) electron density, which is calculated by subtracting ground state observed amplitudes (Fo) from the metastable state Fo (Folight-Fodark), suggests that there is a significant probability that the chromophore is partly in the trans conformation in the metastable state. Ensemble refinement models of both the ground and metastable state structures support disorder of the chromophore 4hydroxybenzylidene ring in the metastable state as compared to the ground state (Figure 3B and D). Residues and waters that line the chromophore cavity are also observed to have structural differences between the ground and metastable state, notably R66, H193 and a water molecule termed W-E. Another important difference between the ground and metastable state structures is the presence or absence of a water (W-A) molecule in the chromophore cavity. Density for W-A is visible in the ground state, but not the metastable state. One explanation for the lack of density for W-A in the metastable state is that movement of the chromophore to the trans isomer displaces this water from the cavity. 
Neutron structure of Dathail ground state. The neutron structure of the ground state of Dathail reveals the chromophore in the cis conformation to be protonated (Figure 4A and B). Neutron density reveals a hydrogen-bonding network from the chromophore 4hydroxybenzylidene ring oxygen to water W-B, which in turn hydrogen bonds to water WC. A surface model of the X-ray ground state structure (Figure 4D) shows that these waters directly connect the chromophore to the bulk solvent through a water channel. The neutron structure also reveals that $\mathrm{H} 193 \mathrm{~N} \varepsilon 2$ is deprotonated in the ground state and accepts a hydrogen bond from the water $\mathrm{W}$-E, whereas $\mathrm{N} \delta 1$ donates a $\mathrm{D}$ atom in a hydrogen bond with E211 (Figure 4C). TGP, Padron, and Dronpa have a glutamate at position144 rather than Dathail's small glycine and W-E in this position, resulting in H193 being coordinated from both sides by large residues in the barrel (E144 and E211) $2,34,35$. As a result, H193 is probably doubly protonated and positively charged in TGP, Padron, and Dronpa, whereas it is singly protonated and neutral in Dathail. In Dronpa, it has been shown that H193 interacts with the 4-hydroxybenzylidene ring of the chromophore through a $\pi$ - $\pi$ stacking interaction, thereby stabilizing the chromophore ${ }^{34} . \pi$ - $\pi$ stacking is similar in Dathail.

\section{Discussion}

The fact that Dathail has two spectrally distinct chromogenic states with such low quantum yields is highly unusual. The photo-induced chromogenic state of Dathail is metastable, with a lifetime of $\sim 50$ min at $20^{\circ} \mathrm{C}$ and $\mathrm{pH} 7.5$ as measured by UV-Vis spectrophotometry, returning back to the ground state through thermal relaxation. Similar proteins, such as Padron and Dronpa, have much longer photo-induced state lifetimes: 14 
$\mathrm{hr}$ and $42 \mathrm{hr}$ respectively ${ }^{36-38}$. In order to understand the origin of these unique properties, we further investigated Dathail in the crystalline state. X-ray crystallography provided structural insights that support a change in conformation and coordination in the chromophore pocket as being responsible for Dathail's photoswitching properties. Neutron crystallography revealed a distribution of hydrogen atoms revealing protonation of the chromophore 4-hydroxybenzylidene group in the ground state. The neutron structure also supports a hypothesis that the photo-induced proton transfer from the chromophore may occur through a water mediated proton relay into the bulk solvent. The structural and mechanistic insights reported here may help in rationally engineering a more diverse population of RSCPs and RSFPs for improved biotechnology and imaging applications.

Although Dathail can be categorized with other proteins as a reversibly photoswitchable GFP-like protein, it is unique in several other ways. First, Dathail was made by mutations that differ drastically from those known to elicit photoswitching in other FPs, such as Padron and Dronpa (Figure 1). Second, Dathail almost lacks green fluorescence in the ground and metastable states, and has extremely low quantum yield in both states ( 0.0034 and 0.0018 respectively), translating into reduced background fluorescence. Third, when Dathail is photoswitched it displays absorbance peaks that are more spectrally separated (108 nm apart), therefore more easily distinguishable, than in similar proteins. This large spectral separation is also observed in other Dathail variants (data not shown). Fourth, Dathail switches $\sim 60$ times more rapidly than Dronpa. Finally, the states can be interchanged for 10 cycles with little loss of spectral properties $(<10 \%)$. The phenotype of Dathail is similar to Padron, but is distinct in the optimal wavelength used for switching to the metastable state ( $405 \mathrm{~nm}$ for Dathail, $488 \mathrm{~nm}$ for Padron) and 
shows more profound spectroscopic differences between the ground and metastable states. In addition Dathail has extremely low fluorescence in both the ground and metastable states, unlike Padron.

Possible mechanistic insights for these properties were revealed in structures determined by X-ray and neutron crystallography. The X-ray crystal structures of Dathail in both the ground and metastable states revealed differences that may be associated with photoswitching. Solving the ground state and metastable state structures of Dathail from crystals grown in the same drop allowed direct structural comparison using isomorphous difference electron density maps. Dathail also provides the perfect test case for ensemble refinement. Although used as the standard in NMR structural biology, multi-model ensembles can now be used in X-ray crystallography, due to recent developments in structure refinement algorithms ${ }^{33,39}$. This approach is particularly valuable as it allows high-resolution, direct comparisons between ordered ground and more disordered metastable states. We found that the chromophore in the ground state is well coordinated through various interactions with surrounding amino acid residues and water, and is in the cis conformation. On the other hand the chromophore in the metastable state is disordered and does not completely adopt either the cis or trans conformations, as was previously observed for Padron ${ }^{35}$. Surrounding residues are also less well ordered. This result strongly suggests that a change in the coordination and conformation of the chromophore is associated with photoswitching. This result is also in agreement with recent FTIR measurements of the M159T mutant of Dronpa, which demonstrated that deprotonation of the 4-hydroxybenzyl ring is mostly responsible for the observed UV-Vis spectral changes occurring upon irradiation ${ }^{40}$. 
However, the X-ray structures alone do not provide insights into the chromogenic properties of Dathail. Indeed, the structure of the Dathail ground state, in particular the chromophore position and its cis conformation, is remarkably similar to those of other FPs. In general, such X-ray structures are associated with fluorescence, yet Dathail is essentially non-fluorescent. We hypothesize that there may be differences in the protonation pattern of the chromophore cavity between Dathail and other FPs that cannot be seen using X-rays. Padron and Dronpa have both been modeled with de-protonated chromophores in their fluorescent, cis states. Although the X-ray structure of the ground state of Dathail was of high quality, we were unable to directly locate the position of hydrogen atoms and therefore to determine the protonation state of the chromophore. The reason for this is that X-rays are scattered by atomic electron clouds, and hydrogen with just one electron is essentially invisible in electron density maps calculated from X-ray data collected above atomic resolution $(\sim 1 \mathrm{~A})$. Even in X-ray structures with atomic resolution, typically only the most ordered hydrogen atoms can be located 41,42 .

Neutrons are strongly sensitive to hydrogen, and can be used to directly visualize hydrogen atoms even at medium resolutions ${ }^{41-44}$. Therefore, neutron crystallography can be used to provide information on the protonation state of the chromophore and surrounding amino acid residues in addition to the coordination of water molecules. However, the determination of neutron structures requires particularly large crystals, which are often difficult to obtain with FPs. Given the enhanced tendency of TGP and its derivatives to crystallize, we were able to obtain a Dathail neutron structure in the ground state, revealing that the chromophore is protonated. The fact that the ground state chromophore of Dathail is clearly protonated and stable with the chromophore in the cis 
conformation (Figure 4A) challenges the consensus view that a stable, coplanar chromophore in the cis isomer leads to stable fluorescence, given that Dathail is essentially non-fluorescent.

Interestingly, the chromophore is connected to the bulk solvent through a hydrogen bonded water chain (waters $\mathrm{W}$-B and $\mathrm{W}-\mathrm{C}$ ), which may ultimately affect the overall resonant state of the chromophore by shuttling excited state protons from the chromophore to bulk solvent. This may explain the loss of fluorescence and the chromogenic nature of the Dathail ground state. This water chain proton relay is absent in other FPs and appears to be formed in Dathail by the mutation of serine to proline at position 142. Perhaps, for robust green fluorescence in the ground state, FPs require a threonine or serine residue at this position. This would allow the deprotonated chromophore 4-hydroxybenzylidene ring to hydrogen bond to the interior of the barrel, preventing protonation by the solvent exposed water channel directly opposite to the phenolic oxygen, as was recently demonstrated for GFP 45 . Further, it may be that if a nonhydrogen-bond-forming residue is present at this position, such as proline in Dathail, protonation and deprotonation of the chromophore 4-hydroxybenzylidene ring becomes possible due to the presence of the water chain connecting the chromophore to bulk solvent. In Dathail, this allows for a protonated chromophore, with blue-shifted absorbance.

Another important observation from the neutron structure is that H193 in the ground state of Dathail is protonated on $\mathrm{N} \delta 1$ and deprotonated on $\mathrm{N} \varepsilon 2$, making it neutral, as expected for a histidine at $\mathrm{pH}$ 8.5. H193 $\pi$-stacks with the 4-hydroxybenzylidene ring of the chromophore, thus providing structural rigidity. The deprotonated $\mathrm{N} \varepsilon 2$ accepts a 
hydrogen bond from a water molecule, and $\mathrm{N} \delta 1$ hydrogen bonds with E211. This protonation state of $\mathrm{H} 193$ is consistent with molecular dynamics simulations of the Padron cis-state. However, in Padron H193 is predicted to hydrogen bond with E144 ${ }^{35}$. The presence of two negatively charged glutamates in hydrogen bonding distance to $\mathrm{H} 193$ would increase its pKa and necessitate it be doubly protonated and positively charged in order to counter the two negative charges on E144 and E211 carboxylic groups. In Dathail, E144 has been mutated to a much smaller glycine and H193 is no longer directly hydrogen bonded to the interior of the $\beta$-barrel, and may be more dynamic. It may be that the amino acid residues at position 144 that coordinate with H193 in TGP, Padron and other FPs anchor the chromophore 4-hydroxybenzylidene in the cis ground state more tightly than H193 is able to do in Dathail. This may further explain the lack of fluorescence in the ground state of Dathail due to a less strongly constrained chromophore. It could also explain why the time required to photoswitch Dathail with $405 \mathrm{~nm}$ and $488 \mathrm{~nm}$ light is much shorter than that of other photoswitching proteins. A less constrained H193 also weakens the constraints on the chromophore ${ }^{3,46}$. In Dathail, it is coordinated by E211, and water W-E (Figure 4), but lacks the additional stabilizing interaction normally afforded by a glutamate at position 144. Even though H193 in Dathail is in the same position as the residue in fluorescent protein counterparts, this loss of coordination of the H193 Nع2 by residue 144 demonstrates how highly precise positioning and coordination of $\mathrm{H} 193$ drastically affects both ground state fluorescence and photoswitching.

On excitation with photoswitching light ( $405 \mathrm{~nm})$, the chromophore region becomes disordered with multiple structural rearrangements. It is important to note that discrete positions for the 4-hydroxybenzylidene and other affected residues could not be reliably 
modeled in the metastable state: either there are discrete states that could not be modeled because those states are short-lived within the time frame of these experiments, or the energy associated with excitation at $405 \mathrm{~nm}$ leads to general disorder within the chromophore cavity that cannot be statically modeled. These movements include those of the 4-hydroxybenzylidene cis region to a variety of positions, including the trans position (Figure 3).

As a result of the 4-hydroxybenzylidene movement, water molecule, $\mathrm{W}-\mathrm{A}$, which resides in the trans region of the ground state chromophore, is bumped out of place (Figure 3A-D). H193 is no longer constrained by the $\pi$-stacking and acquires many conformations. These conformations change the distances between H193, E211, and water molecule W-E, in turn altering the interaction with the chromophore. Furthermore, as the 4hydroxybenzylidene swings towards the trans region, and loses interaction with H193, R66 re-localizes to the region above the trans chromophore, possibly interacting with the 4hydroxybenzylidene in a cation- $\pi$ interaction in the metastable state (Figure 3E-H). M146 also moves slightly away from the chromophore cavity, to allow for the change in R66 position. Other residues in the chromophore vicinity, such as V157, Y177 and E211, also show disorder after excitation, supporting this notion of 4-hydroxybenzyl ring movement. In addition, many waters that are carefully coordinated by these residues in the ground state (waters W-E, W-B, W-F, W-G, and others not named but visible in the structure figures) are disrupted by the drastic change in chromophore cavity interactions and occupation.

The value of using extremely stable fluorescent proteins as scaffolds for libraries of mutations was first shown for sfGFP ${ }^{47}$, where the fluorescence of the stable variant (sfGFP) 
was shown to be far more resistant to mutation than the FP (eGFP) from which it was derived. With TGP, we demonstrate that not only does stability confer resistance to mutation, but also that it is likely to yield novel proteins that would not otherwise be found in less stable scaffolds. This is illustrated not only by the isolation of Dathail, but also the variety of similar photoswitching variants obtained from these libraries, many of which have additional interesting properties and potential applications. One exciting example is Glas, a variant isolated from the photoswitching library that is fluorescent in the ground state, but photoswitches to an entirely dark metastable state similar to the metastable state of Dathail. Glas and Dronpa are similar in overall sequence homology (78\% identity), but accommodate the trans isomerization of the 4-hydroxybenzylidene group through the modification of entirely different residues, demonstrating the enormous combinatorial potential for further engineering. Characterization and exploration of Glas and other unique mutants from the libraries described in this work will result in greater mechanistic understanding, and greater use in a wider variety of applications. This is particularly true for Glas, due to its retention of S142, but lack of E144 (mutated to serine). The significance of S142 has been long known, but a role for E144 in fluorescence and switching has been underappreciated until now.

Beyond the spectroscopic curiosity of exhibiting significant absorption without fluorescence, in both spectrally switchable states, Dathail has several characteristics that are improvements in terms of applications. These include low background fluorescence, large spectral separation, rapid switching time and the ability to switch states many times. These performance enhancements mean Dathail is likely to be extremely useful in the quickly developing fields of imaging and biosensors, including pcFRET, high resolution 
microscopy, and live tracking within the cell. Beyond these potential uses, however, the utility of Dathail's unusual spectral properties remain unexplored, and with further experimentation are likely to lead to presently unimagined uses.

\section{Methods}

\section{Libraries of variants using eCGP123 as scaffold}

Primers were designed to create fragments of the eCGP gene that included NNK degeneracy. Fragments were gel purified, and then assembled using DNA assembly. DNA assembly was mediated by a minimum of 20 nucleic acid overlaps. Assemblies of each library were gel purified, restriction digested, and ligated into an expression vector (pETCK3), transformed into BL21, and sequenced. This method produced large enough libraries to cover the theoretical diversity of the mutations introduced. Libraries were expressed by plating and induction with IPTG (1mM). Libraries were stored after harvesting, by freezing resuspended colonies in $10 \%$ glycerol. In each evolutionary step, clones of interest served as templates for the next round of evolution by colony PCR, allowing for the gradual and iterative addition of more mutations.

Ratiometric flow cytometry was used to screen for clones expressing red-shifted variants of eCGP123 in plated and expressed samples of each library. A BD FACS Aria flow cytometer was used for ratiometric sorting. Ratios were plotted on scatter plots based on different combinations of the following filters: 505/10, and 525/10, and later, 530/30, with excitation of 488nm. Events were gated first by fluorescence in the 525/10 fluorescence channel and then by ratio of $525 / 10: 505 / 10$, or $530 / 30: 505 / 10$. Bacteria which appeared in the sort gate with a high ratio of 500-510nm signal: 520-530nm signal, and 
also had a significant fluorescence in the 520-530nm wavelength were collected. 500 events were collected for each sample, unless the population was too small to yield this number. Controls included E. coli with no transformed plasmids, and E.coli expressing eCGP123. Sorted populations were plated onto 2XYT Kanamycin glucose agar plates (KG plates) the surface of which had been covered with nitrocellulose membranes before streaking. Plates were incubated at $37^{\circ} \mathrm{C}$ for about 16 hours, and then induced by transferring the nitrocellulose membranes onto the surface of 2XYT Kanamycin IPTG agar plates (KI plates). Induced plates were then incubated at $30^{\circ} \mathrm{C}$ for 4 hours, before storing at $18^{\circ} \mathrm{C}$.

Using a 488nm illuminator, clones from the sorted population expressing protein emitting red fluorescence were picked. These variants were further grown and expressed and excitation and emission spectra were obtained from induced culture. The clones expressing the most red-shifted protein were used as template for the next round of evolution.

\section{Libraries of photoswitching variants using TGP}

Although initial evolution was carried out using eCGP123, mutations were transferred to TGP as soon as this protein had been developed. TGP did not aggregate like eCGP123, and was also slightly more thermostable. A TGP library was produced using assembly PCR and degenerate primers to introduce desired mutations. More than covering the theoretical size, 480 clones were randomly picked, grown in 96-well format, and sequenced.

Sequencing data were used to identify each possible variant. Photoswitching clones were identified by imaging of induced plated libraries in ambient light and with a fluorescence 
channel. After exposure to $405 \mathrm{~nm}$ wavelength light, plates were re-imaged. Images were compared, and variants selected for further characterization

\section{Purification of variants}

Protein samples were purified in $1 \mathrm{~L}$ preps. Plates were streaked out from the glycerol stocks using sterile inoculation loops onto 2XYT Kanamycin glucose agar plates (KG plates) the surface of which had been covered with nitrocellulose membranes before streaking. Plates were incubated at $37^{\circ} \mathrm{C}$ for about 16 hours, and then induced by transferring the nitrocellulose membranes onto the surface of 2XYT Kanamycin IPTG agar plates (KI plates). Induced plates were then incubated at $30^{\circ} \mathrm{C}$ for $4 \mathrm{hrs}$, before storing at $18^{\circ} \mathrm{C}$.

$5 \mathrm{ml}$ overnight cultures were incubated at $37^{\circ} \mathrm{C}$, shaking. $1 \mathrm{~L}$ (in a $2.5 \mathrm{~L}$ baffled Erlenmeyer flask) of 2XTY/Kan media was inoculated with $1 \mathrm{ml}$ of overnight culture. They were grown at $37^{\circ} \mathrm{C}$, or until optical density at $600 \mathrm{~nm}$ reaches 0.8 a.u. Cultures were induced with $1 \mathrm{mM} \mathrm{IPTG}$, and the temperature reduced to $30^{\circ} \mathrm{C}$ for four hours. After 4 hours, the temperature was reduced to $16^{\circ} \mathrm{C}$ and induction continued for about 72 hours.

Cells were harvested by centrifugation. Pellets were resuspended in $35 \mathrm{ml}$ of lysis buffer (25 $\mathrm{mM} \mathrm{Na}_{2} \mathrm{PO}_{4}, 25 \mathrm{mM} \mathrm{Na}_{2} \mathrm{HPO}_{4}, 10 \mathrm{mM}$ imidazole, $300 \mathrm{mM} \mathrm{NaCl} \mathrm{pH7).} \mathrm{Each}$ resuspended pellet was then lysed by running through a high pressure Canadian press (from Avestin, with pressure of 25,000) psi until fluid cycled through 5 times. The lysate was then centrifuged in $50 \mathrm{ml}$ tubes at $4{ }^{\circ} \mathrm{C}$ for 30 minutes, at $14500 \mathrm{rpm}$. The cell pellet was discarded, and lysate supernatant pressed through 0.45-micron syringe filters. Phosphate buffer solution (PBS) was then used to equilibrate $10 \mathrm{ml}$ Nickel NTA Agarose by centrifuging nickel NTA solution at 800rpm for five minutes, discarding supernatant and 
replacing supernatant with PBS thrice, for three total washes. Five ml resin was resuspended in PBS to filtered lysate, and incubated, rotating in a cold room.

Affinity chromatography was performed with $15 \mathrm{ml}$ plastic columns. Lysate elution and resin incubate was poured into the column so that non-bound protein and solution flowed freely out of the column. A fraction of this was kept for later analysis. The column and resin were washed twice with $25 \mathrm{ml}$ of lysis buffer, and once with wash buffer $(25 \mathrm{mM}$ $\mathrm{Na}_{2} \mathrm{PO}_{4}, 25 \mathrm{mM} \mathrm{Na}_{2} \mathrm{HPO}_{4}, 20 \mathrm{mM}$ imidazole, $300 \mathrm{mM} \mathrm{NaCl} \mathrm{pH} \mathrm{7).} \mathrm{Protein} \mathrm{was} \mathrm{fully} \mathrm{eluted}$ from the resin by adding $7 \mathrm{ml}$ of Elution buffer $\left(25 \mathrm{mM} \mathrm{Na}_{2} \mathrm{PO}_{4}, 25 \mathrm{mM} \mathrm{Na}_{2} \mathrm{HPO}_{4}, 250 \mathrm{mM}\right.$ imidazole, $300 \mathrm{mM} \mathrm{NaCl} \mathrm{pH} \mathrm{7)} \mathrm{to} \mathrm{the} \mathrm{column.}$

Protein eluate was concentrated to a volume of $3 \mathrm{ml}$ or less. Concentrating tubes were centrifuged for 5 minutes, eluate removed, and concentrated protein mixed in the upper portion of the tube. This was repeated until protein volume less than $3 \mathrm{ml}$. Concentrated proteins were then each further purified using size exclusion chromatography on the AKTA prime system and a HighLoad 26/60 Superdex 200 prepgrade $320 \mathrm{ml}$ column. The column was equilibrated with TNG buffer at a rate of 1.5 $\mathrm{ml} /$ minute. Three $\mathrm{ml}$ fractions were collected at a rate of $(1 \mathrm{ml} / \mathrm{min})$. UV absorbance correlating to fractions was inspected, and fractions with a higher UV absorbance than background were collected and analyzed by SDS polyacrylamide gel electrophoresis.

Samples were further diluted to $122 \mathrm{mM}$ final concentration for spectroscopy experiments and aliquoted into $20 \mathrm{ul}$ aliquots. These aliquots were flash frozen in liquid nitrogen and then stored at -80 degrees. 
Dathail protein for crystallography was prepared in the same manner, but concentrated to $65-90 \mathrm{mg} / \mathrm{ml}$ for crystallization. An additional purification step was also required, as optimization of crystallization conditions revealed that Dathail protein with no his-tag grew crystals with improved diffraction. Therefore, Dathail was expressed with a TEV cleavage site linker to the His-tag. Protein was expressed as indicated above, however after affinity chromatography, the eluate was concentrated to $4 \mathrm{ml}$, and then combined with purified TEV protein. The reaction was dialyzed overnight at $16^{\circ} \mathrm{C}$, in $5 \mathrm{ml}$ dialysis cassettes (Thermo Scientific Slide-A-Lyzer, 3.5K MWCO ) into 4L of lysis buffer (50mM Na2P04, 50mM Na2HPO4, 10mM imidazole, $100 \mathrm{mM} \mathrm{NaCl}$ ). The reaction was removed from the dialysis cassettes and loaded onto a plastic column with $10 \mathrm{ml}$ Nickel NTA Agarose (prepared as indicated above). The column was washed with lysis buffer and eluate was collected, until the eluate was no longer tinted orange. The sample was then concentrated, and further purified using size exclusion chromatography, as described above.

\section{Fluorescence spectroscopy}

Fluorescence spectra were collected using a Horiba Jobin Yvon Fluoromax 4 spectrofluorometer, with an excitation/emission band pass of $2 \mathrm{~nm}$. Quantum yields were determined by the comparative gradient method, with quinine sulfate $\left(0.1 \mathrm{M} \mathrm{H}_{2} \mathrm{SO}_{4}\right)$ and fluorescein $(0.1 \mathrm{M} \mathrm{NaOH})$ as selected standards because of their close absorbance/fluorescence spectral match with the ground and metastable states, respectively. At $\rrbracket_{\mathrm{exc}}=370 \mathrm{~nm}$ and $\bigoplus_{\mathrm{exc}}=493 \mathrm{~nm}$, quinine sulfate and fluorescein have established quantum yields of 0.54 and 0.92 , respectively, at the adopted conditions. The estimated uncertainties are less than $10 \%$ for quantum yield determinations.

\section{Photoswitching experiments (Dathail)}


The photoinduced conversion between protein states was performed upon continuous irradiation at $\square_{\max }=389 \mathrm{~nm}$ (ground to metastable state) and $?_{\max }=497 \mathrm{~nm}$ (metastable to ground state). These experiments were facilitated by exposure of samples to light within the same spectrofluorometer as above (Horiba Jobin Yvon Fluoromax 4), using its kinetics mode to control the excitation wavelength and monitor fluorescence decay at the peak wavelength. The power density of incident light was $0.2 \mathrm{~mW} / \mathrm{cm}^{2}$ at the sample $\left(?_{\mathrm{exc}}=389\right.$ or $497 \mathrm{~nm}$, band pass $\left.=5 \mathrm{~nm}\right)$. The conversion was fully reversible (as shown by the cycling tests) and complete after about $1 \mathrm{~min}$ and $5 \mathrm{~min}$, respectively, for the forward (ground to metastable state) and backward (metastable to ground state) processes. For these measurements, the sample was prepared at a concentration of $11 \mu \mathrm{M}$ (Abs $<0.2$ at ? $\left.?_{\max }\right)$.

\section{Fluorescence lifetime measurements}

In lifetime measurements, the spectrofluorometer was coupled with a timecorrelated single photon counting (TCSPC) system from Horiba Jobin Yvon. The apparatus was equipped with a pulsed laser diode source (NanoLED) operating at $1 \mathrm{MHz}$ and with excitation centered at 390 or $455 \mathrm{~nm}$, depending on the state of interest. Each measurement was terminated when a maximum peak preset of 20,000 photon counts was reached for the monitored fluorescence. Analyses of fluorescence decay profiles were performed with the Horiba DAS6 software.

\section{X-ray Crystal tray setup- Sitting drops}

Five hundred ul of crystallization condition (0.2M Magnesium Chloride, 0.1M BisTris:HCl pH 6.5, 25\% PEG 3350) was added to the well of a Hampton crystallography 24well non-greased tray, and a sitting drop bridge was dropped into the well. The drop was 
added to the bridge well and mixed with a small volume (in differing ratios) of the well solution. The chambers were sealed with tape. Trays were labeled, covered with the lid and incubated in a Styrofoam box inside an incubator of desired temperature $(6,10,16,18$, or $\left.20^{\circ} \mathrm{C}\right)$.

\section{X-ray structure determination with individual and ensemble refinement}

Crystals were grown, optimized, and tested by X-ray diffraction. Data was collected on two crystals from the same drop from the condition: 0.2M Magnesium Chloride, $0.1 \mathrm{M}$ Bis-Tris: $\mathrm{HCl} \mathrm{pH} \mathrm{6.5,} \mathrm{25 \%} \mathrm{PEG} \mathrm{3350.} \mathrm{Spectrally} \mathrm{different} \mathrm{states} \mathrm{between} \mathrm{the} \mathrm{two} \mathrm{crystals}$ were obtained by collecting diffraction data on one crystal with minimal exposure to light, and collecting diffraction data on the second crystal while exposing the crystal to $405 \mathrm{~nm}$ wavelength light from a 50mW laser, before cryo-cooling, and during data collection at 100K. Structures of Dathail in the ground state and in the metastable state were solved to 1.65 Å resolution. The data sets were recorded using HKL-3000 on a Rigaku highflux home source using a wavelength of $1.54178 \AA$, and integrated scaled and merged using iMosflm and Scala (within the ccp4 suite) software. Structures were determined using the molecular replacement program Phenix.MR with a single TGP molecule as a search model, followed by refinement using the program Phenix.refine. A single Dathail molecule in the asymmetric unit was identified and refined. Iterative model building and inspection between rounds of refinement was performed using Coot and graphics were displayed using Pymol software. Dathail mutations were modeled at appropriate locations and simulated annealing was performed in the first few rounds of refinement to remove phase bias introduced from the TGP search model. The data collection and refinement statistics are given in Table 2. 


\section{Neutron crystal data collection}

\section{Data collection.}

Neutron time-of-flight Laue diffraction data were collected at 293K on the macromolecular neutron diffractometer MaNDi 4849 located at the Spallation Neutron Source (Oak Ridge National Laboratory, Oak Ridge, USA). A total of 10 crystal settings were collected to a maximum usable resolution of $2.5 \AA$. Each exposure was 24 hours long and used neutrons with wavelengths between the range of $2-4 \AA$. Between each exposure a $\varphi$ step of $10^{\circ}$ was carried out. As is usual for a Laue experiment, the crystal was held stationary at a different $\varphi$ setting for each exposure. The diffraction images were processed using Mantid software 50 and scaled for detector response using the Argonne National Laboratory variable wavelength data reduction program (Andrev) ${ }^{51}$. The resulting reflection file was examined and merged with XPREP (a program from the SHELXTL suite). Monochromatic roomtemperature X-ray diffraction data were collected using a Rigaku HomeFlux system, equipped with a MicroMax-007 HF generator and Osmic VariMax optics. The diffraction images were obtained using a RAXIS-IV++ image-plate detector. Diffraction data were collected, integrated and scaled using HKL3000 software suite ${ }^{52}$. The room-temperature X-ray structure of Dathail was refined using $C N S 5353,54$ at the resolution of $2.1 \AA$ before using it as a starting model in joint $\mathrm{X}$-ray/neutron refinement $(\mathrm{XN})^{4}$. A summary of the experimental and refinement statistics is given in Table 1.

Crystal trays were setup with $50 \mathrm{ml}$ of the following crystallization condition: $0.2 \mathrm{M}$ Sodium Acetate, 22\% (weight/ volume) Polyethylene glycol 4,000, 0.1 M Tris:HCl pH 8.5. In order to perform joint X-ray/neutron refinement of the data collected, an X-ray structure must be 
collected on a crystal in the same condition and environment. Therefore, multiple large crystals were mounted from the trays from which the $10 \mathrm{~mm}^{3}$ crystal (see below) was collected. The crystals were mounted in capillaries and exchanged with optimized Hydrogen/ Deuterium exchange buffer (25\% PEG4000, and 0.2 M NaOac in $\mathrm{D}_{2} \mathrm{O}$ ). After exchanging for a minimum of 7 days, crystals were screened on a home-source, Rigaku HomeFlux system at the CSMB at ORNL. Data were collected on a plate-like crystal, which diffracted well in a test shot. 100 frames were collected with 120 -second exposures, with a sample to detector distance of $130 \mathrm{~mm}$. Though beam time was awarded at multiple institutions, the Dathail neutron structure was successfully collected on the MaNDi diffractometer at ORNL ${ }^{49}$. A $10 \mathrm{~mm}^{3}$ plate-like crystal from the condition $0.2 \mathrm{M}$ Sodium Acetate, 22\% weight/volume Polyethylene glycol 4,000, 0.1 M Tris:HCl pH 8.5 and Hydrogen/Deuterium exchange buffer (25\% PEG4000, and 0.2 M NaOac in $\mathrm{D}_{2} \mathrm{O}$ ) was mounted and exchanged in a short capillary. We used a total of 10 crystal orientations. At each orientation, the crystal was kept stationary as time-of-flight (TOF) Laue images were collected to a maximum resolution of $2.5 \AA$. The data were collected and images were processed and scaled. Refinement was performed using nCNS ${ }^{55}$ and manual model building in Coot ${ }^{56}$ with relevant joint X-ray/neutron refinement statistics listed in Table 4.

\section{Joint XN structure refinement.}

The joint XN structure of Dathail was determined using $n C N S^{55}$ and manipulated in $\operatorname{Coot}^{56}$. After initial rigid-body refinement, several macrocycles of positional, atomic displacement parameter, and occupancy refinement followed. Between each macrocycle the structure 
was checked, side-chain conformations were altered and water molecule orientations were built based on the $\mathrm{F}_{0}-\mathrm{F}_{\mathrm{C}}$ difference and $2 \mathrm{~F}_{0}-\mathrm{F}_{\mathrm{C}}$ neutron scattering length density map. The $2 \mathrm{~F}_{0}-\mathrm{F}_{\mathrm{C}}$ and $\mathrm{F}_{\mathrm{O}}-\mathrm{F}_{\mathrm{C}}$ neutron scattering density maps were then examined to determine the correct orientation of hydroxyl (Ser, Thr, Tyr) and ammonium (Lys) groups, and protonation states of His and Lys residues. The protonation states of some disordered side chains could not be obtained directly, and remained ambiguous. All water molecules were refined as $\mathrm{D}_{2} \mathrm{O}$. Initially, water oxygen atoms were positioned according to their electron density peaks, and then were shifted slightly in accordance with the neutron scattering length density maps. The levels of $\mathrm{H} / \mathrm{D}$ exchange at $\mathrm{OH}, \mathrm{NH}$ and $\mathrm{SH}$ sites were refined. All labile H positions in Dathail were modeled as D and then the occupancy of D was allowed to refine within the range of -0.56 to 1.00 (the scattering length of $\mathrm{H}$ is -0.56 times the scattering length of D). Before depositing the final structure to the PDB, a script was run that converts a record for the coordinate of $\mathrm{D}$ atom into two records corresponding to an $\mathrm{H}$ and a D atom partially occupying the same site, both with positive partial occupancies that add up to unity. The Ramachandran statistics for the Dathail structure reported here are as follows: residues in most favored regions $95.7 \%$, residues in additional allowed regions $4.3 \%$.

PDB accession codes:

Dathail ground state X-ray structure: 5EB6

Dathail metastable state X-ray structure: 5EB7

Dathail ground state joint X-ray/neutron structure: 5EBJ

Dathail ground state X-ray ensemble refinement: 5EJU 
Dathail metastable state X-ray ensemble refinement: 5EXU

Acknowledgements

This research at ORNL's Spallation Neutron Source was sponsored by the Scientific User Facilities Division, Office of Basic Energy Sciences, U.S. Department of Energy. The Office of Biological and Environmental Research supported research at Oak Ridge National Laboratory's Center for Structural Molecular Biology (CSMB), using facilities supported by the Scientific User Facilities Division, Office of Basic Energy Sciences, U.S. Department of Energy.

\section{Figures}

Figure 1. A. X-ray structure of eCGP123 with mutations made to create libraries shown as sticks. B. Barrel view of the libraries. C. Bacterial colonies grown on an agar plate expressing different variants of a library. D. Bacterial colonies on an agar plate, expressing eCGP123. E. Sequence alignment of TGP, Dathail, Phanta, Dronpa, and Padron. Mutations that were made on the eCGP123 scaffold to create libraries are indicated with asterices of different colors. Colors of asterices align with the colored residues indicated in $1 \mathrm{~A}$ and $1 \mathrm{~B}$.

Figure 2. A. Schematics of reversibly photoswitchable proteins Dathail, Dronpa, and Padron, their photoswitching wavelengths, and quantum yields of both states. B. Ground 
state and light-induced-state absorbance spectra of Dathail. C. Dathail forward and reverse photoswitching kinetics.

Figure 3. A. Ground state X-ray structure 2Fo-Fc, chromophore region. B. Ground state ensemble refinement, chromophore region. C. Light-induced state X-ray structure $2 \mathrm{Fo}-\mathrm{Fc}$, chromophore region. D. Light-induced state X-ray structure ensemble refinement, chromophore region. E. Isomorphous difference density map, (ground state Fo)-(lightinduced state Fo). F. Ground state X-ray structure 2Fo-Fc, upper chromophore region. G. Ground state ensemble refinement, upper chromophore region. H. Light induced state Xray structure $2 \mathrm{Fo}-\mathrm{Fc}$, upper chromophore region. I. Light-induced state X-ray structure ensemble refinement, upper chromophore region. J. Isomorphous difference density map, (ground state Fo)-(Light-induced state Fo).

Figure 4. A. Joint X-ray/Neutron structure of Dathail, chromophore region with X-ray 2FoFc (teal) and neutron 2Fo-Fc (red) mesh of sigma level 1.5. B. Neutron 2Fo-Fc mesh (red) with sigma level of 1, water channel. C. Joint X-ray/Neutron structure of Dathail upper chromophore region, with X-ray 2Fo-Fc (teal), and neutron 2Fo-Fc (red) mesh of sigma level 1.5. D. Surface model of X-ray ground state, with difference density map, (ground state Fo)-(Light-induced state Fo).

Supplementary Figure 1. A. Ground state and light-induced-metastable state excitation and emission spectra of Dathail. B. Dathail fluorescence emission during multiple cycles of 
photoswitching and relaxation.

A Dathail excitation and emission spectra

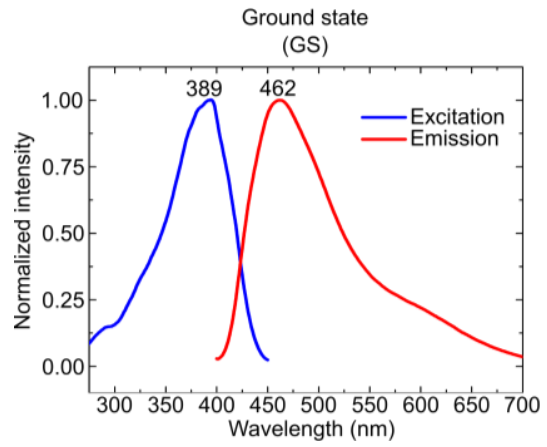

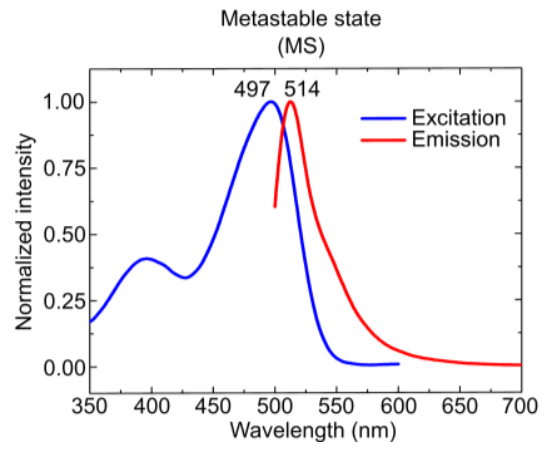

B Dathail reversibility

GS fluorescence decay upon excitation at $389 \mathrm{~nm}$

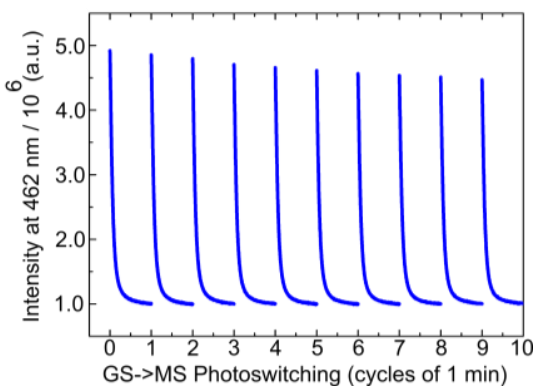

Supplementary Figure 2. Dronpa forward and reverse photoswitching kinetics.

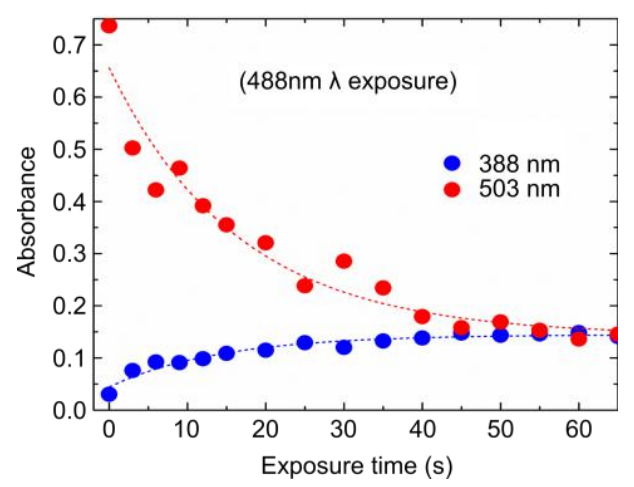

Schematic 1.

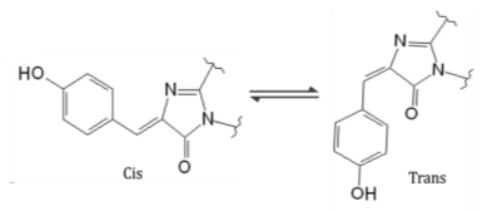

Table 1. Lifetimes of the photo-induced state of Dathail as a function of temperature and $\mathrm{pH}$

\begin{tabular}{|c|c|c|c|c|}
\hline $\begin{array}{c}\text { Temperature } \\
(\mathrm{C})\end{array}$ & $\begin{array}{c}\text { Life-time } \\
\text { (min) } \mathrm{pH} \\
=10\end{array}$ & $\begin{array}{c}\text { Life-time } \\
(\mathrm{min}) \mathrm{pH}= \\
8.5\end{array}$ & $\begin{array}{c}\text { Life-time } \\
(\mathrm{min}) \mathrm{pH}= \\
7.5\end{array}$ & $\begin{array}{c}\text { Life-time } \\
(\mathrm{min}) \mathrm{pH}= \\
6\end{array}$ \\
\hline 10 & 430 & 390 & 154 & 590 \\
\hline 20 & 114 & 114 & 53 & 180 \\
\hline
\end{tabular}




\begin{tabular}{|c|c|c|c|c|}
30 & 33 & 38 & 18 & 55 \\
\hline 40 & 10 & 12 & 7 & 17 \\
\hline 50 & 3.4 & 4 & 2.5 & 5 \\
\hline 60 & - & 1.4 & 0.9 & - \\
\hline
\end{tabular}

Table 2. X-ray crystallographic data collection and refinement statistics.

\begin{tabular}{|c|c|c|c|c|}
\hline & \multicolumn{2}{|l|}{$\begin{array}{l}\text { Dathail } \\
\text { ground state }\end{array}$} & \multicolumn{2}{|l|}{$\begin{array}{l}\text { Dathail } \\
\text { metastable state }\end{array}$} \\
\hline \multicolumn{5}{|l|}{ Data collection } \\
\hline Space group & \multicolumn{2}{|l|}{ P 21212} & \multicolumn{2}{|l|}{ P 21212} \\
\hline \multicolumn{5}{|l|}{ Cell dimensions } \\
\hline$a, b, c(\AA)$ & \multicolumn{2}{|l|}{$\begin{array}{l}75.97,81.09 \\
39.44\end{array}$} & \multicolumn{2}{|l|}{$\begin{array}{l}76.25,81.18 \\
47.90\end{array}$} \\
\hline$\alpha, \beta, \gamma\left({ }^{\circ}\right)$ & \multicolumn{2}{|l|}{$90,90,90$} & \multicolumn{2}{|l|}{$90,90,90$} \\
\hline Resolution $(\AA ̊)$ & \multicolumn{2}{|l|}{$28.27-1.65$} & \multicolumn{2}{|l|}{$32.18-1.65$} \\
\hline No. unique reflections & \multicolumn{2}{|l|}{$29380(2586)$} & \multicolumn{2}{|l|}{$29829(2624)$} \\
\hline$R_{\text {sym }}$ or $R_{\text {merge }}$ & \multicolumn{2}{|l|}{$0.044(0.212)$} & \multicolumn{2}{|l|}{$0.063(0.304)$} \\
\hline$I / \sigma I$ & \multicolumn{2}{|l|}{$18.08(4.09)$} & \multicolumn{2}{|l|}{$10.91(2.18)$} \\
\hline Completeness (\%) & \multicolumn{2}{|l|}{$97.7(87.3)$} & \multicolumn{2}{|l|}{$98.6(88.1)$} \\
\hline Redundancy & \multicolumn{2}{|l|}{$5.5(4.1)$} & \multicolumn{2}{|l|}{$5.1(3.9)$} \\
\hline \multirow[t]{2}{*}{ Refinement } & Single model & Ensemble & Single Model & Ensemble \\
\hline & Refinement & Refinement & Refinement & Refinement \\
\hline No. models & 1 & 47 & 1 & 63 \\
\hline Resolution $(\AA ̊)$ & $28.27-1.65$ & $28.27-1.65$ & $32.18-1.65$ & $32.18-1.65$ \\
\hline$R_{\text {work }} / R_{\text {free }}$ & $0.159 / 0.198$ & $0.152 / 0.194$ & $0.183 / 0.217$ & $0.168 / 0.210$ \\
\hline \multicolumn{5}{|l|}{ No. atoms } \\
\hline Protein & 1729 & 80887 & 1721 & 104981 \\
\hline Ligand/ion & 24 & 1128 & 24 & 1464 \\
\hline Water & 269 & 7271 & 283 & 9062 \\
\hline \multicolumn{5}{|l|}{$B$-factors } \\
\hline Protein & 33.90 & & 31.70 & \\
\hline Ligand/ion & 25.30 & & 36.50 & \\
\hline Water & 41.90 & & 39.90 & \\
\hline \multicolumn{5}{|l|}{ R.m.s. deviations* } \\
\hline Bond lengths $(\AA)$ & 0.010 & 0.019 & 0.006 & 0.016 \\
\hline Bond angles $\left({ }^{\circ}\right)$ & 1.48 & 2.155 & 1.24 & 1.705 \\
\hline \multicolumn{5}{|l|}{ Ramachandran } \\
\hline Favored (\%) & 99.0 & & 99.0 & \\
\hline Outliers (\%) & 0 & & 0 & \\
\hline
\end{tabular}

Table 3. Room temperature neutron crystallographic data collection and joint XN refinement statistics.

\begin{tabular}{ll}
\hline & Dathail \\
\hline Data collection & \\
Beamline/Facility & $\mathrm{MaNDi}$ \\
Space group & $\mathrm{P} 2{ }_{1} 2{ }_{1}$ \\
Cell dimensions & \\
$a, b, c(\AA)$ & $77.21,82.05,40.89$ \\
$\quad \alpha, \beta, \gamma\left({ }^{\circ}\right)$ & $90,90,90$
\end{tabular}




\begin{tabular}{ll} 
Resolution $(\AA)$ & $40.00-2.50(2.56-$ \\
& $2.50)^{*}$ \\
No. reflections unique & $6168(273)$ \\
$R_{\text {merge }}$ & $0.317(0.263)$ \\
$I / \sigma I$ & $3.9(2.2)$ \\
Completeness (\%) & $63.6(47.9)$ \\
Redundancy & $1.4(0.8)$ \\
Data rejection criteria & no observation \& \\
& $|\mathrm{F}|=0$ \\
& \\
Joint XN Refinement & \\
Resolution (neutron, $\AA)$ & $40-2.50$ \\
Resolution (X-ray, $\AA$ ) & $40-2.1$ \\
Sigma cut-off & 2.5 \\
No. reflections (neutron) & 5652 \\
No. reflections (X-ray) & 11102 \\
$R_{\text {work }} / R_{\text {free }}($ neutron) & $0.305 / 0.344$ \\
$R_{\text {work }} / R_{\text {free }}(\mathrm{X}-$ ray) & $0.234 / 0.252$ \\
No. atoms & \\
Protein including H and D & 3433 \\
Water & $276\left(92 \mathrm{D}_{2} \mathrm{Os}\right)$ \\
$B$-factors & \\
Protein & 42.9 \\
Water & 50.3 \\
R.m.s. deviations & \\
Bond lengths $(\AA)$ & 0.010 \\
Bond angles ( $)$ & 1.276 \\
\hline
\end{tabular}

\section{References}

1. Kiss, C., Temirov, J., Chasteen, L., Waldo, G. S. \& Bradbury, A. R. M. Directed evolution of an extremely stable fluorescent protein. Protein Eng. Des. Sel. 22, 313-323 (2009).

2. Close, D. W. et al. TGP, an extremely stable, non-aggregating fluorescent protein created by structure-guided surface engineering. Proteins Struct. Funct. Bioinforma. n/a-n/a (2014). doi:10.1002/prot.24699

3. Pakhomov, A. A. \& Martynov, V. I. GFP Family: Structural Insights into Spectral Tuning. Chem. Biol. 15, 755-764 (2008). 
4. Dedecker, P., De Schryver, F. C. \& Hofkens, J. Fluorescent Proteins: Shine on, You Crazy Diamond. J. Am. Chem. Soc. 135, 2387-2402 (2013).

5. Stepanenko, I. A. et al. in Lat 2010 Int. Conf. Lasers Appl. Technol. (eds. Panchenko, V., Mourou, G. \& Zheltikov, A. M.) 7994, (2011).

6. Chudakov, D. M., Matz, M. V., Lukyanov, S. \& Lukyanov, K. A. Fluorescent Proteins and Their Applications in Imaging Living Cells and Tissues. Physiol. Rev. 90, 1103-1163 (2010).

7. Shimomura, O., Johnson, F. H. \& Saiga, Y. Extraction, Purification and Properties of Aequorin, a Bioluminescent Protein from the Luminous Hydromedusan, Aequorea. J. Cell. Comp. Physiol. 59, 223-239 (1962).

8. Yang, F., Moss, L. G. \& Phillips Jr, G. N. The molecular structure of green fluorescent protein. Dept Biochem. Cell Biol. Rice Univ. (1997).

9. Ormö, M. et al. Crystal Structure of the Aequorea victoria Green Fluorescent Protein. Science 273, 1392-1395 (1996).

10. Cubitt, A. B. et al. Understanding, improving and using green fluorescent proteins. Trends Biochem. Sci. 20, 448 - 455 (1995).

11. Lukyanov, K. A. et al. Natural Animal Coloration Can Be Determined by a Nonfluorescent Green Fluorescent Protein Homolog. J. Biol. Chem. 275, 25879-25882 (2000).

12. Chudakov, D. M., Feofanov, A. V., Mudrik, N. N., Lukyanov, S. \& Lukyanov, K. A. Chromophore environment provides clue to 'kindling fluorescent protein' riddle. J. Biol. Chem. 278, 7215-7219 (2003). 
13. Habuchi, S. et al. Reversible single-molecule photoswitching in the GFP-like fluorescent protein Dronpa. Proc. Natl. Acad. Sci. U. S. A. 102, 9511-9516 (2005).

14. Dedecker, P., Flors, C., Hotta, J., Uji-i, H. \& Hofkens, J. 3D Nanoscopy: Bringing Biological Nanostructures into Sharp Focus. Angew. Chem. Int. Ed. 46, 8330-8332 (2007).

15. Tanabe, T. et al. Multiphoton excitation-evoked chromophore-assisted laser inactivation using green fluorescent protein. Nat. Methods 2, 503-505 (2005).

16. Subach, F. V. et al. Photoactivation mechanism of PAmCherry based on crystal structures of the protein in the dark and fluorescent states. Proc. Natl. Acad. Sci. U. S. A. 106, 21097-21102 (2009).

17. Lummer, M. et al. Reversible photoswitchable DRONPA-s monitors nucleocytoplasmic transport of an RNA-binding protein in transgenic plants. Traffic Cph. Den. 12, 693-702 (2011).

18. Andresen, M. et al. Structural basis for reversible photoswitching in Dronpa. Proc. Natl. Acad. Sci. 104, 13005-13009 (2007).

19. Jung, G. Fluorescent Proteins. (Springer, 2012).

20. Shkrob, M. A., Mishin, A. S., Chudakov, D. M., Labas, Y. A. \& Lukyanov, K. A. Chromoproteins of the green fluorescent protein family: Properties and applications. Russ. J. Bioorganic Chem. 34, 517-525 (2008).

21. Verkhusha, V. V. \& Lukyanov, K. A. The molecular properties and applications of Anthozoa fluorescent proteins and chromoproteins. Nat Biotech 22, 289-296 (2004).

22. Ganesan, S., Ameer-beg, S. M., Ng, T. T. C., Vojnovic, B. \& Wouters, F. S. A dark yellow fluorescent protein (YFP)-based Resonance Energy-Accepting Chromoprotein (REACh) 
for Förster resonance energy transfer with GFP. Proc. Natl. Acad. Sci. U. S. A. 103, 40894094 (2006).

23. Pettikiriarachchi, A., Gong, L., Perugini, M. A., Devenish, R. J. \& Prescott, M. Ultramarine, a Chromoprotein Acceptor for Forster Resonance Energy Transfer. Plos One 7, (2012).

24. Cho, S. et al. Simple super-resolution live-cell imaging based on diffusion-assisted Förster resonance energy transfer. Sci. Rep. 3, (2013).

25. Don Paul, C. et al. Phanta: A Non-Fluorescent Photochromic Acceptor for pcFRET. PLoS ONE 8, e75835 (2013).

26. Zhou, X. X. \& Lin, M. Z. Photoswitchable fluorescent proteins: ten years of colorful chemistry and exciting applications. Curr. Opin. Chem. Biol. 17, 682 - 690 (2013).

27. Dai, M. et al. The creation of a novel fluorescent protein by guided consensus engineering. Protein Eng. Des. Sel. 20, 69-79 (2007).

28. Brakemann, T. et al. Molecular Basis of the Light-driven Switching of the Photochromic Fluorescent Protein Padron. J. Biol. Chem. 285, 14603-14609 (2010).

29. Brakemann, T. et al. A reversibly photoswitchable GFP-like protein with fluorescence excitation decoupled from switching. Nat. Biotechnol. 29, 942-U132 (2011).

30. Grotjohann, T. et al. Diffraction-unlimited all-optical imaging and writing with a photochromic GFP. Nature 478, 204-208 (2011).

31. Grotjohann, T. et al. rsEGFP2 enables fast RESOLFT nanoscopy of living cells. eLife 1, (2012).

32. Doublié, S. Macromolecular Crystallography Protocols: Structure Determination. (Springer Science \& Business Media, 2006). 
33. Burnley, B. T., Afonine, P. V., Adams, P. D. \& Gros, P. Modelling dynamics in protein crystal structures by ensemble refinement. eLife 1, e00311-e00311 (2012).

34. Mizuno, H. et al. Light-dependent regulation of structural flexibility in a photochromic fluorescent protein. Proc. Natl. Acad. Sci. 105, 9227-9232 (2008).

35. Faro, A. R. et al. Low-temperature chromophore isomerization reveals the photoswitching mechanism of the fluorescent protein Padron. J. Am. Chem. Soc. 133, 16362-16365 (2011).

36. Yadav, D. et al. Real-time monitoring of chromophore isomerization and deprotonation during the photoactivation of the fluorescent protein Dronpa. J. Phys. Chem. B 119, 2404-2414 (2015).

37. Ando, R., Mizuno, H. \& Miyawaki, A. Regulated Fast Nucleocytoplasmic Shuttling Observed by Reversible Protein Highlighting. Science 306, 1370-1373 (2004).

38. Tinnefeld, P., Eggeling, C. \& Hell, S. W. Far-Field Optical Nanoscopy. (Springer, 2015).

39. Adams, P. D. et al. PHENIX : a comprehensive Python-based system for macromolecular structure solution. Acta Crystallogr. D Biol. Crystallogr. 66, 213-221 (2010).

40. Kaucikas, M., Tros, M. \& van Thor, J. J. Photoisomerization and proton transfer in the forward and reverse photoswitching of the fast-switching M159T mutant of the Dronpa fluorescent protein. J. Phys. Chem. B 119, 2350-2362 (2015).

41. Blakeley, M. P., Langan, P., Niimura, N. \& Podjarny, A. Neutron crystallography: opportunities, challenges, and limitations. Curr. Opin. Struct. Biol. 18, 593-600 (2008).

42. Langan, P. \& Chen, J. C.-H. Seeing the chemistry in biology with neutron crystallography. Phys Chem Chem Phys 15, 13705-13712 (2013). 
43. Bennett, B. C. \& Yeager, M. The lighter side of a sweet reaction. Structure 18, 657-659 (2010).

44. Kovalevsky, A. Y. et al. Identification of the Elusive Hydronium Ion Exchanging Roles with a Proton in an Enzyme at Lower pH Values. Angew. Chem. Int. Ed. 50, 7520-7523 (2011).

45. Laptenok, S. P. et al. Complete Proton Transfer Cycle in GFP and Its T203V and S205V Mutants. Angew. Chem. Int. Ed Engl. (2015). doi:10.1002/anie.201503672

46. Prescott, Mark et al. In preparation.

47. Pedelacq, J.-D., Cabantous, S., Tran, T., Terwilliger, T. C. \& Waldo, G. S. Engineering and characterization of a superfolder green fluorescent protein. Nat Biotech $\mathbf{2 4}, \mathbf{7 9 - 8 8}$ (2006).

48. Coates, L. et al. The Macromolecular Neutron Diffractometer MaNDi at the Spallation Neutron Source. J Appl Cryst 48, 1302-1306 (2015).

49. Coates, L., Stoica, A. D., Hoffmann, C., Richards, J. \& Cooper, R. The macromolecular neutron diffractometer (MaNDi) at the Spallation Neutron Source, Oak Ridge: enhanced optics design, high-resolution neutron detectors and simulated diffraction. J. Appl. Crystallogr. 43, 570-577 (2010).

50. Draper, N. The Mantid Project: Notes from an International Software.

51. Schultz, A. J., Srinivasan, K., Teller, R. G., Williams, J. M. \& Lukehart, C. M. Single-crystal, time-of-flight, neutron-diffraction structure of hydrogen cisdiacetyltetracarbonylrhenate, [cis-(OC)4Re(CH3CO)2] H: a metallaacetylacetone molecule. J. Am. Chem. Soc. 106, 999-1003 (1984). 
52. Minor, W., Cymborowski, M., Otwinowski, Z. \& Chruszcz, M. HKL -3000: the integration of data reduction and structure solution - from diffraction images to an initial model in minutes. Acta Crystallogr. D Biol. Crystallogr. 62, 859-866 (2006).

53. Brünger, A. T. et al. Crystallography \&amp; NMR System: A New Software Suite for Macromolecular Structure Determination. Acta Crystallogr. D Biol. Crystallogr. 54, 905921 (1998).

54. Brunger, A. T. Version 1.2 of the Crystallography and NMR system. Nat. Protoc. 2, 27282733 (2007).

55. Adams, P. D., Mustyakimov, M., Afonine, P. V. \& Langan, P. Generalized X-ray and neutron crystallographic analysis: more accurate and complete structures for biological macromolecules. Acta Crystallogr. D Biol. Crystallogr. 65, 567-573 (2009).

56. Emsley, P., Lohkamp, B., Scott, W. G. \& Cowtan, K. Features and development of Coot. Acta Crystallogr. D Biol. Crystallogr. 66, 486-501 (2010). 
Figure 1

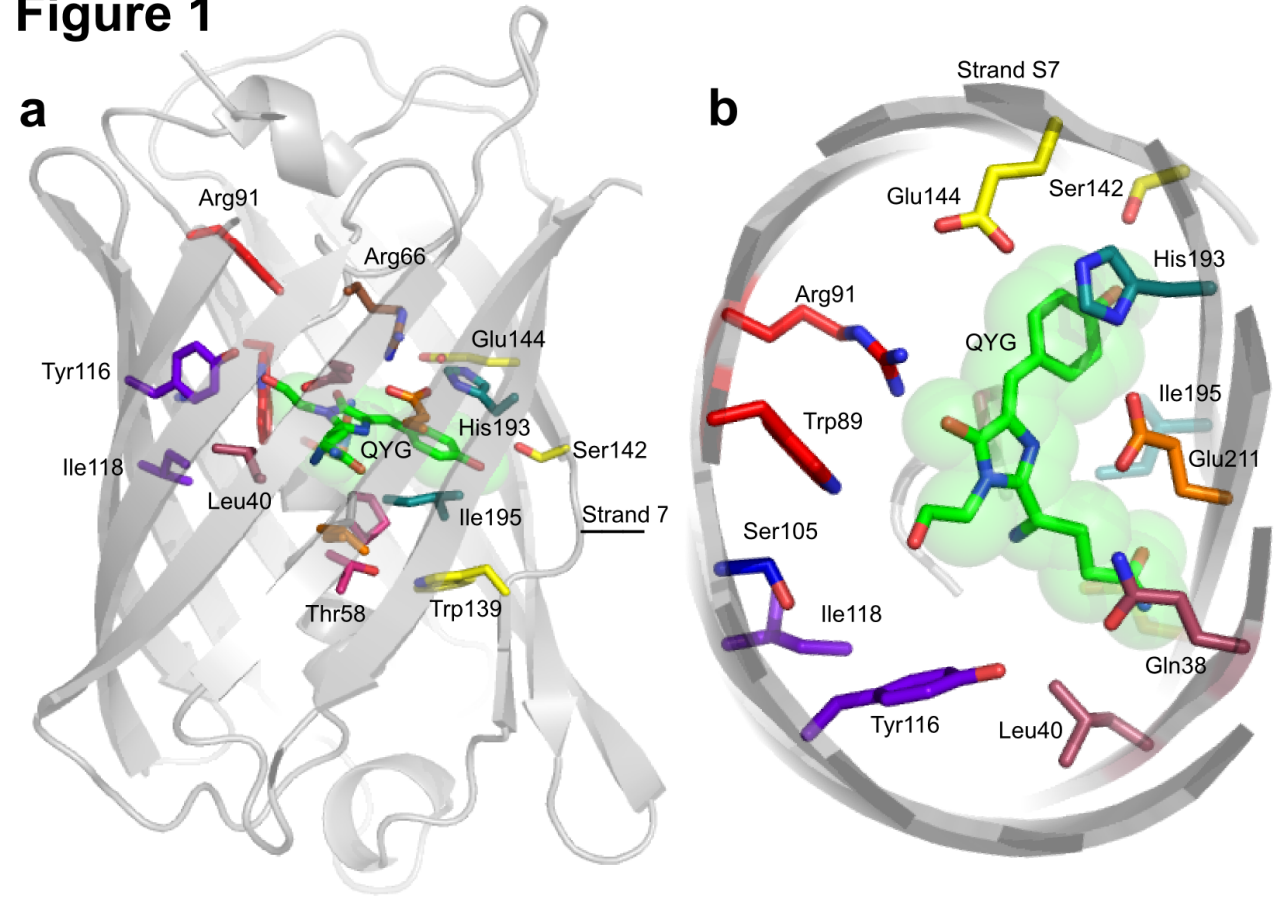

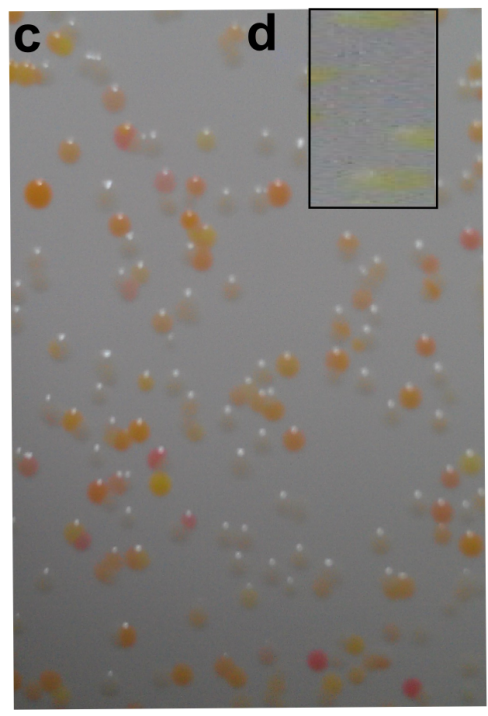

e

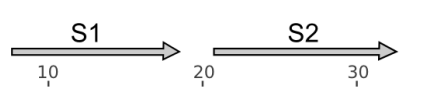

$\underset{40}{\stackrel{S 3}{\longrightarrow}}$

H1 $c h r$.

H2

$\stackrel{\mathrm{S} 4}{90}$

$\longrightarrow$

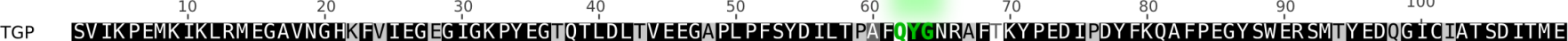

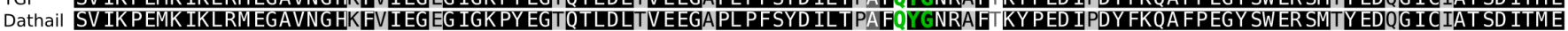

Phanta SV IKPEMK IKLRMEGAVNGHKFV IEGEG IGK PYEGTQTLDLTVKEGAPLPFSYD ILTPAFMYGNRAFVKY PKD IPDYFKQAFPEGY SWER SMTYEDQ G IC IAT SD ITME

Dronpa SVIKPDMK IKLRMEGAVNGHPFAIEGVGLGKPFEGKQSMDLKVKEGGPLPFAYD ILTTVECIGNRVEAKYPENIVDYFKQSFPEGY SWER SMNYEDGG ICNATND ITLD

Padron SVIKPDMKIKLRMEGAVNGHPEAIEGVGLGKPFEGKQSMDLKVKEGG PLPFAYD ILTIMAFCEANRVIFAKYPEN IVDYFKQSFPEGYSWERSMIYEDGG ICNATNDITLD

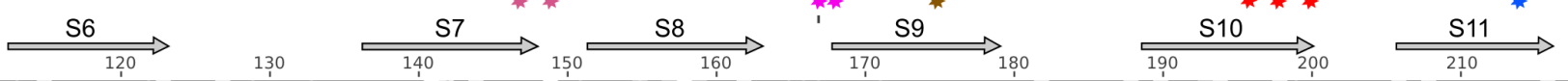

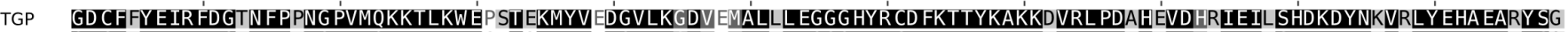

Dathail GDCFFYEIRFDGTNFPPNGPVMQKKTLKWKSPTGKMYVEDGVLKGDVEMALLLEGGGHYRCDFKTTYKAKKDVRLPDAHEVDHRIEILSHDKDYNKVRLYEHAEARY SG

Phanta GDCFFYK IRFDGTNFP PNG PVMOKKTLKWEPSTEKMYVR DGVLKGDVN MALLLEGGGHYRCDFKTTYKAKKDVRLPDAHEVDQR IEILSHDKDYNKVRLYEHAEARYSM

Dronpa GDCYIYEIRFDGVNFPANGPVMQKRTVKWEPSTEKLYVRDGVLKGDVN MALSLEGGGHYRCDFKTTYKAKKVVQLPDYHFVDHHIEIKSHDKDYSNVNLHEHAEA-HSE

Dronpa GDCYIYEIRFDGVNFPANGPVMQKRTVKWEPSTEKLYVRDGVLKGDVNMALSLEGGGHYRCDFKTTYKAKKVVQLPDYHFVDHHIEIKSHDKDYSNVNLHEHAEA-HSE
Padron GDCYIYEIRFDGVNFPANGPVMQKRTVKWELSTEKLYVRDGVLKSDGNYALSLEGGGHYRCDFKTTYKAKKVVQLPDYHSVDHHIEIKSHDKDYSNVNLHEHAEA-HS

*- sites at which NNK diversity introduced in color libraries. 


\section{Figure 2}

a

Ground state

(GS)
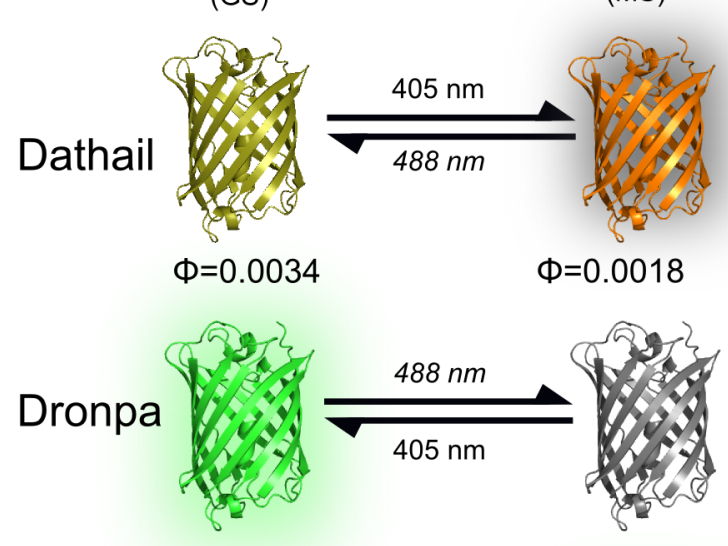

$\Phi=0.68$

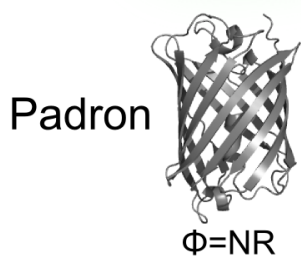

Metastable state $\mathbf{b}$

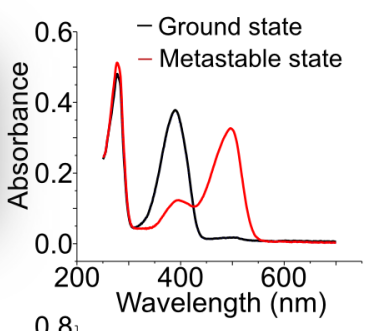

0.8
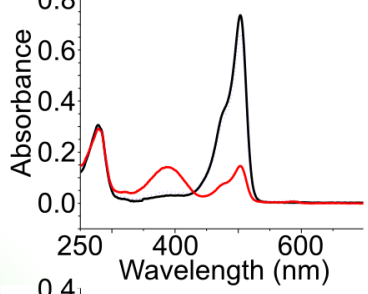

0.4 Wavelength $(\mathrm{nm})$

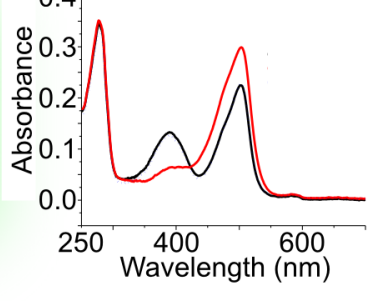

Dathail switching kinetics
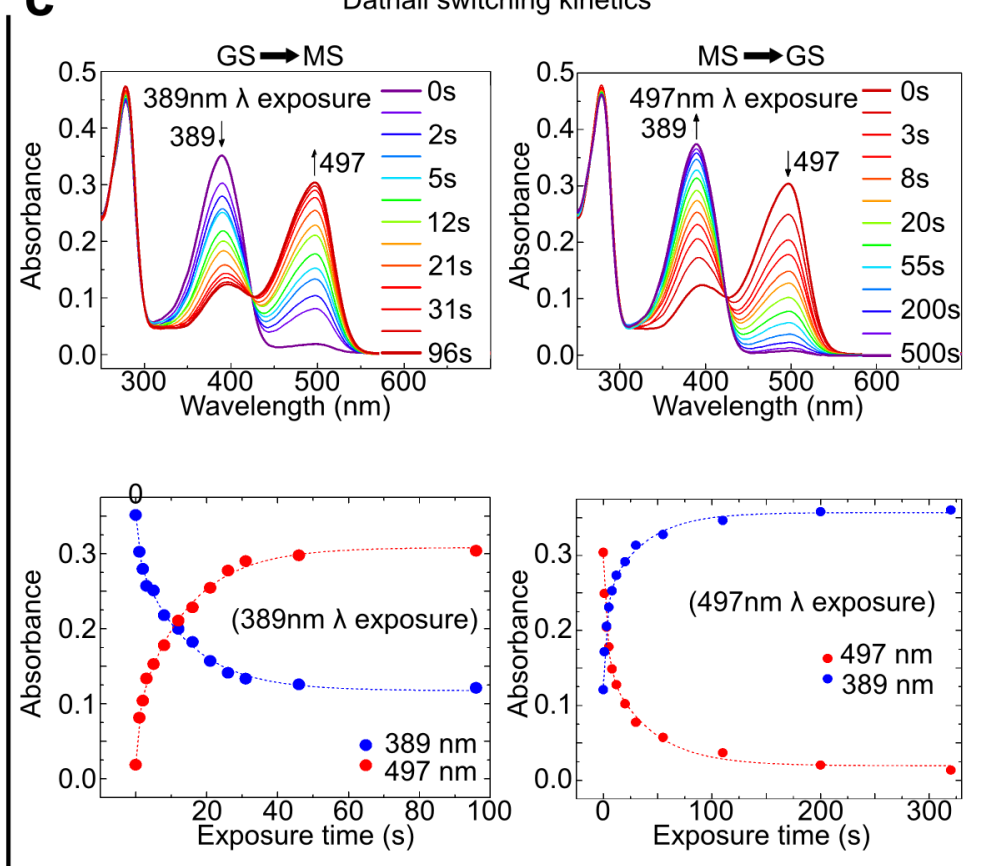
Figure 3

Figure 3

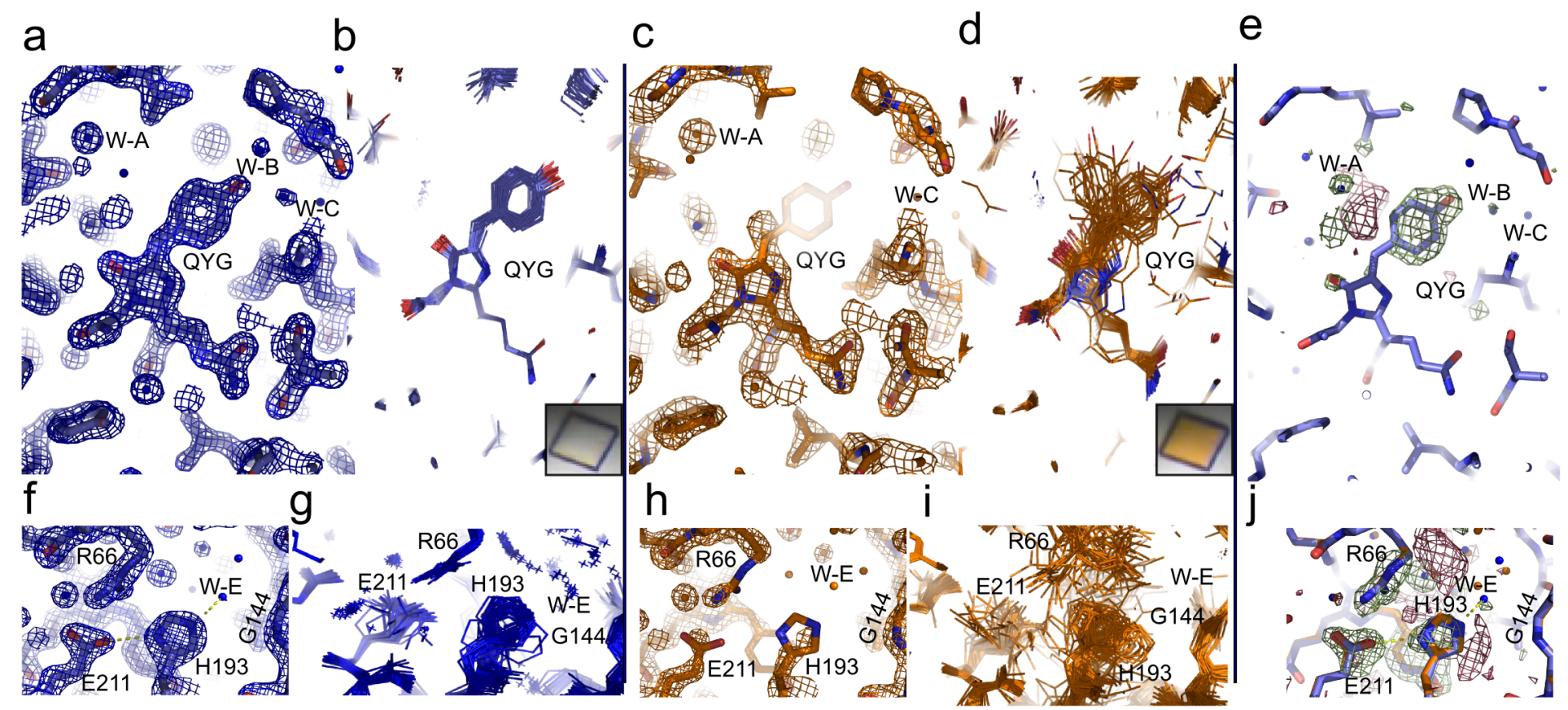




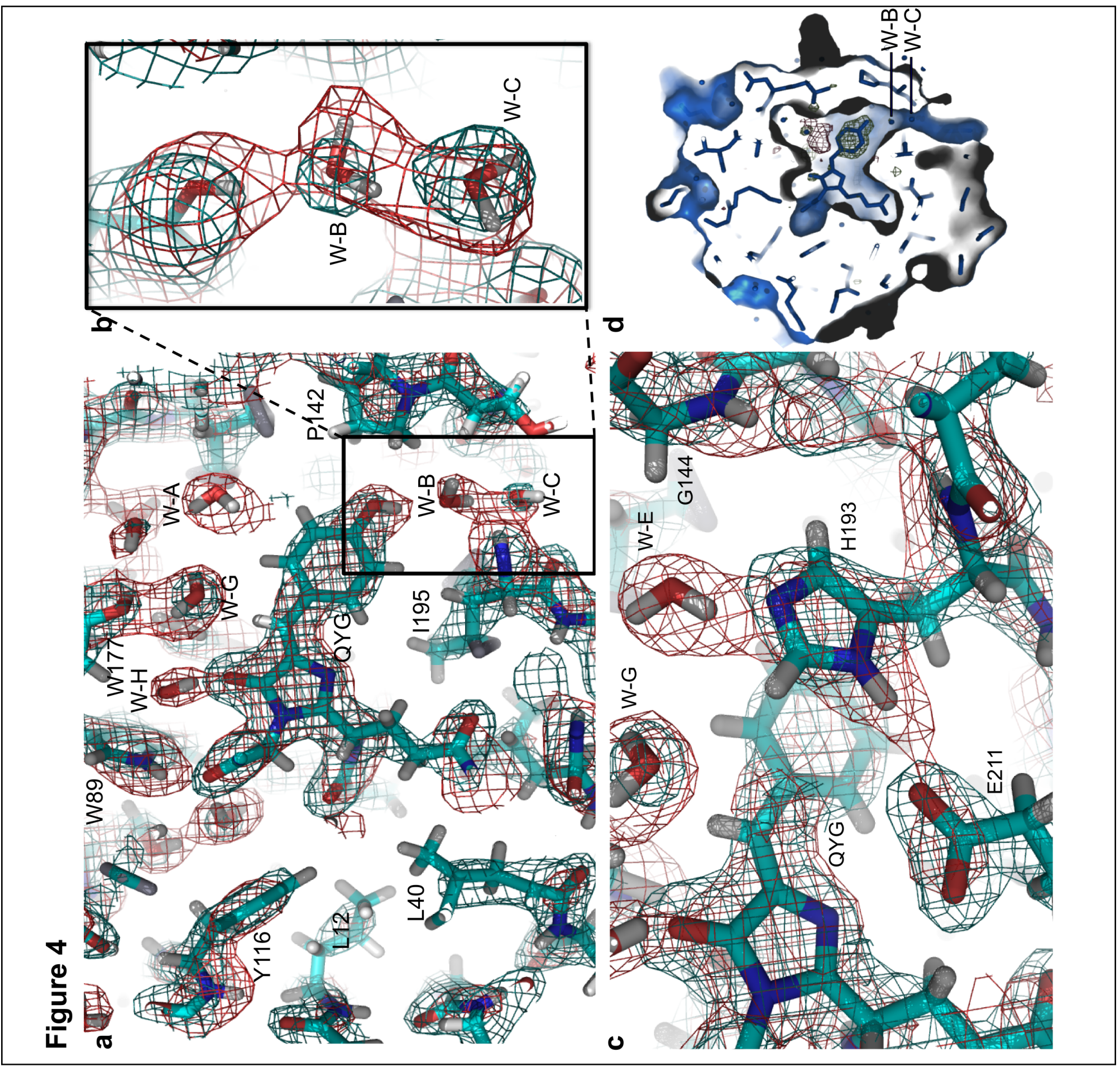




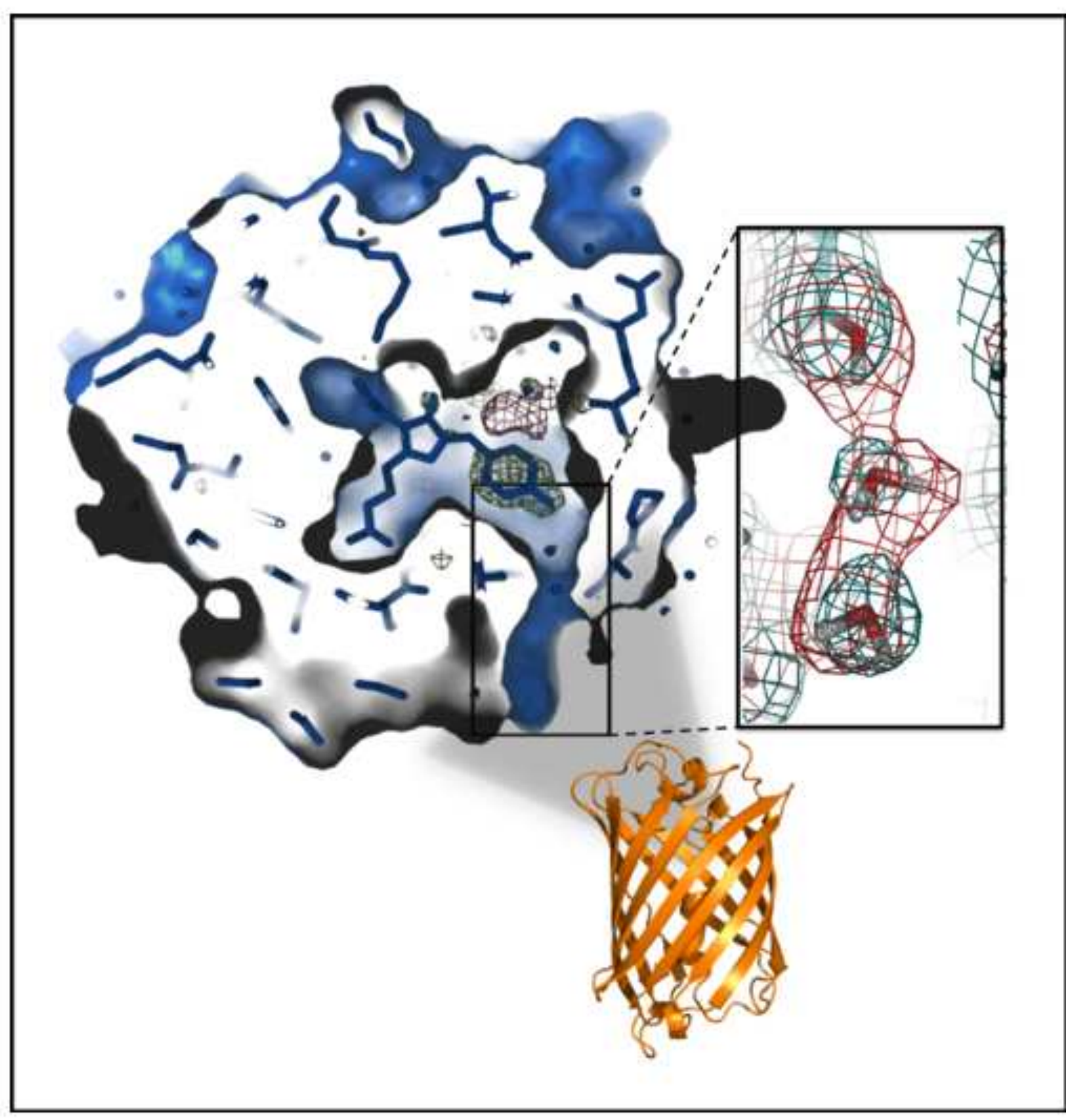

